\section{Muerte súbita por fibrilación ventricular en deportista. Una presentación inusual del Síndrome de Brugada. Caso Clínico}

\author{
JOSÉ PARDO G. ${ }^{1}$, JULIÁN SEPÚLVEDA T. ${ }^{1}$, GONZALO LIRA LP. ${ }^{1}$, \\ ARMANDO PÉREZ S. ${ }^{2}$, BRUNO DIGHERO E. ${ }^{3}$, \\ MATÍAS HERRERA F. ${ }^{3}$, FRANCISCA GONZÁLEZ A. ${ }^{3}$, \\ JORGE EGGER Z. ${ }^{4}$, GABRIELA RAMÍREZ L. ${ }^{4}$, \\ ALAN WIENER D. ${ }^{5}$, MARÍA LORENA JOFRÉ SM. ${ }^{6}$
}

\section{Sudden cardiac death during exercise due to Brugada syndrome. Report of one case}

We report a 20-year-old male athlete who while running, presented a sudden death due to ventricular fibrillation. He was successfully rescued by cardiopulmonary resuscitation maneuvers and an automatic external defibrillator. Without evidence of structural heart disease, Brugada syndrome was diagnosed as the cause, after which a subcutaneous implantable cardioverter defibrillator was indicated. We discuss the subject of sudden cardiac death in athletes and its unusual relationship with exercise in this channelopathy.

(Rev Med Chile 2021; 149: 939-944)

Key words: Brugada Syndrome; Death, Sudden, Cardiac; Exercise.
'Unidad de Arritmias y Laboratorio de Electrofisiología Cardíaca, Hospital Militar de Santiago. Santiago, Chile. ¿Unidad de Arritmias, Hospital Regional de Concepción. Concepción, Chile. ${ }^{3} U$ nidad Coronaria, Hospital Militar de Santiago. Santiago, Chile.

${ }^{4}$ Servicio de Anestesiología, Hospital Militar de Santiago. Santiago, Chile. ${ }^{5}$ Servicio de Medicina, Hospital Militar de Santiago. Santiago, Chile.

${ }^{6}$ Unidad de Hemodinamia, Hospital Militar de Santiago. Santiago, Chile.

Trabajo no recibió financiamiento. Los autores declaran no tener conflictos de interés.

Recibido el 30 de diciembre de 2020 , aceptado el 30 de junio de 2021 .

Correspondencia a:

Dr. José Pardo G.

Unidad de Arritmias y Laboratorio de Electrofisiología Cardíaca. Hospital Militar de Santiago. Av. Fernando Castillo Velasco 9100, La Reina. Santiago, Chile. joseapardo@hotmail.com
E n los Estados Unidos de América, la muerte súbita (MS) extrahospitalaria con una incidencia superior a 350.000 casos/año, presenta una sobrevida inferior a $10 \%$, siendo fundamental para aumentarla, el reconocimiento precoz del paro cardiorespiratorio e inmediato inicio de maniobras de reanimación cardiopulmonar básica (RCPB) con aplicación de desfibrilador externo automático (DEA) ${ }^{1}$.

La MS cardíaca (MSC) ocurre en forma natural, rápida e inesperada, con pérdida de conciencia dentro de la primera hora de iniciados los síntomas, en un sujeto con o sin cardiopatía conocida preexistente $^{2}$.

Atleta es aquel joven o adulto, aficionado o profesional, que realiza ejercicio en forma regular participando en competencias deportivas ${ }^{3}$.

En el atleta joven la MSC es infrecuente, trágica y devastadora, constituyendo la primera causa de muerte. Con una prevalencia de un caso en 50.000 atletas, es más frecuente en hombres, durante ejercicio habitualmente recreacional y con testigos, siendo la fibrilación ventricular (FV) la arritmia más frecuente, con escasa sobrevida ${ }^{4}$.

En menores de 35 años, la miocardiopatía hipertrófica, la displasia arritmogénica de ventrículo derecho y las anomalías congénitas de las arterias coronarias son las principales causas de MSC, y en menor frecuencia las canalopatías sin cardiopatía estructural $(<5 \%)$, dentro de las cuales, el síndrome de Brugada ( $\mathrm{SBr}$ ) es responsable de un caso en 2.000-5.000 atletas jóvenes ${ }^{5-7}$.

La taquicardia ventricular polimorfa (TVP) característica del $\mathrm{SBr}$, habitualmente ocurre en reposo, en el sueño nocturno asociada a vagotonía ${ }^{8}$, siendo excepcional su presentación durante actividad física ${ }^{9}$, como también la posibilidad de ser detectada y tratada exitosamente por DEA, 
dispositivo eficaz en estas arritmias ${ }^{10}$. Recuperada la víctima, se indica el desfibrilador automático implantable (DAI) para prevención secundaria de $\mathrm{MSC}^{2}$.

\section{Historia clínica}

Se presenta el caso de un paciente varón de 20 años de edad, sin antecedentes mórbidos personales ni familiares de importancia. Atleta competitivo con 8 horas de práctica deportiva a la semana, mientras corría los 800 metros sufrió paro cardiorrespiratorio sin aviso, recibiendo inmediatamente maniobras de RCPB y aplicación de DEA en primeros 5 min de ocurrido el evento. Exitosamente reanimado, evolucionó sin secuelas, con hemodinamia estable, en ritmo sinusal, sin apremio respiratorio. Ingresó a UCO Hospital Militar. Al examen, despierto, sin recordar lo ocurrido, en buena condición general, afebril, presión arterial 136/80, frecuencia cardíaca 109 x min, y respiratoria $16 \times$ min, saturación $96 \%$ con $\mathrm{FIO}_{2}$ ambiental. Auscultación cardíaca ritmo regular en 2 tiempos, sin soplos. Resto normal. ECG y radiografía $(\mathrm{Rx})$ tórax sin alteraciones. Laboratorio: $\mathrm{pH} 7,57, \mathrm{pCO}_{2} 20,6 \mathrm{~mm} / \mathrm{Hg}, \mathrm{pO}_{2} 88,4 \mathrm{~mm} / \mathrm{Hg}$, HC03 20,3 mmol/l; leucocitos 13.000; CK total 853 (VN: 46-171 u/l), CK-MB 2,68 (VN 0-4,99 $\mathrm{ng} / \mathrm{ml})$; Troponina ultrasensible 52,5 $(\mathrm{VN}<45,43$ $\mathrm{pg} / \mathrm{ml})$. Sin detección plasmática de cannabinoide THC ni cocaína. PCR COVID-19 negativo. Test
Holter de arritmias mostró escasa extrasistolía supra y ventricular. Ecocardiografía bidimensional, cardioresonancia nuclear magnética, cinecoronariografía, tomografía axial computarizada cerebral y electroencefalograma resultaron normales. Sin evidencia de cardiopatía estructural, el análisis retrospectivo del ECG mostró imagen de bloqueo incompleto de rama derecha (BIRD) en V1 (Figura 1A) tomado a los 70 min post descarga del DEA. Un nuevo ECG con derivaciones precordiales de V1 a V6 ubicadas en segundo, tercer y cuarto espacio intercostal mostró patrón Brugada (pBr) tipo III en V1-V2 (Figura 1B). La prueba con Ajmalina ev a los 5 min, mostró viraje a pBr tipo I en V1-V2 (Figura 1C). Interrogado el DEA modelo HeartStart HS1 Philips, se registró fibrilación ventricular (FV), su desfibrilación con 150 Joules $(\mathrm{J})$ y posterior asistolía, seguida de ritmo ventricular durante RCPB y finalmente ritmo sinusal (Figura $2 \mathrm{~A}, \mathrm{~B}$ y C). El 6 de octubre 2020 bajo anestesia general, implantamos DAI SBc Emblem Boston Scientific, que se observa en $\mathrm{Rx}$ tórax en proyecciones anteroposterior (AP) y lateral (Lat) (Figura $3 \mathrm{~A}, \mathrm{~B})$. Alta 15 de octubre en buenas condiciones, asintomático cardiovascular, con restricción de actividad deportiva competitiva y de contacto, con recomendaciones sugeridas en www.brugada.org.

Test genético para SBr negativo, resultado que no descarta su presencia. Se obtuvo consentimiento informado del paciente para su publicación.
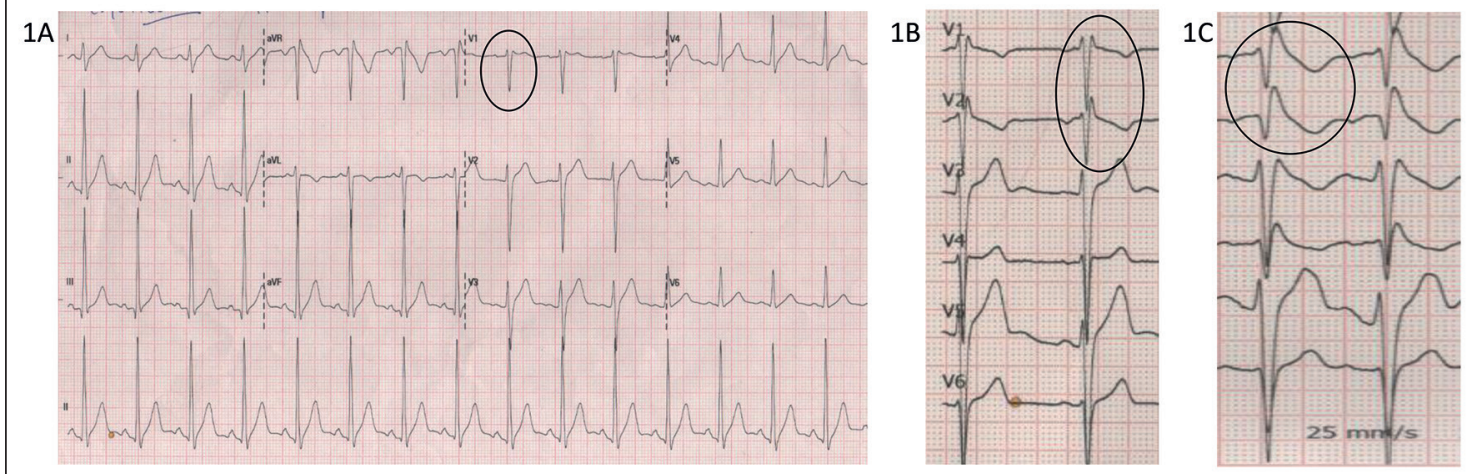

Figura 1. ECG basal y con derivaciones precordiales V1 a V6 registradas en II, III y IV espacio intercostal pre y post administración Ajmalina ev. A: ECG basal registrado en ubicación clásica. Círculo en V1 muestra imagen de BIRD. B: ECG con derivaciones precordiales altas V1-V2-V3. Círculo en V1-V2 muestra pBr tipo III pre-administración Ajmalina. C: ECG con derivaciones precordiales altas V1-V2-V3. Círculo en V1-V2 muestra pBr tipo I, 5 minutos post-administración Ajmalina. 


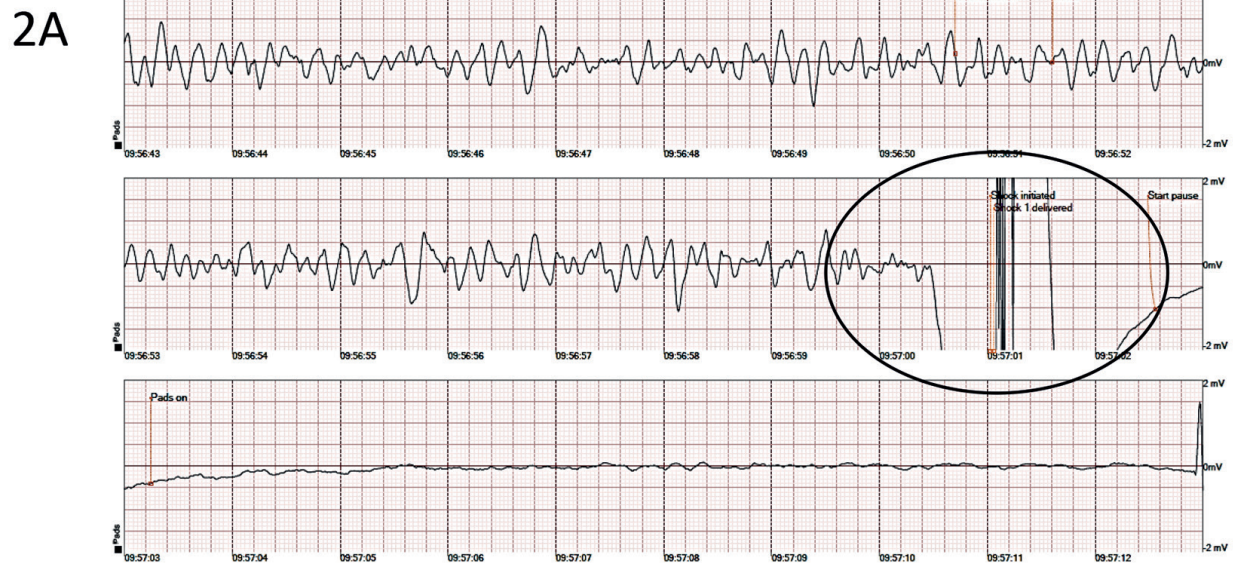

2B
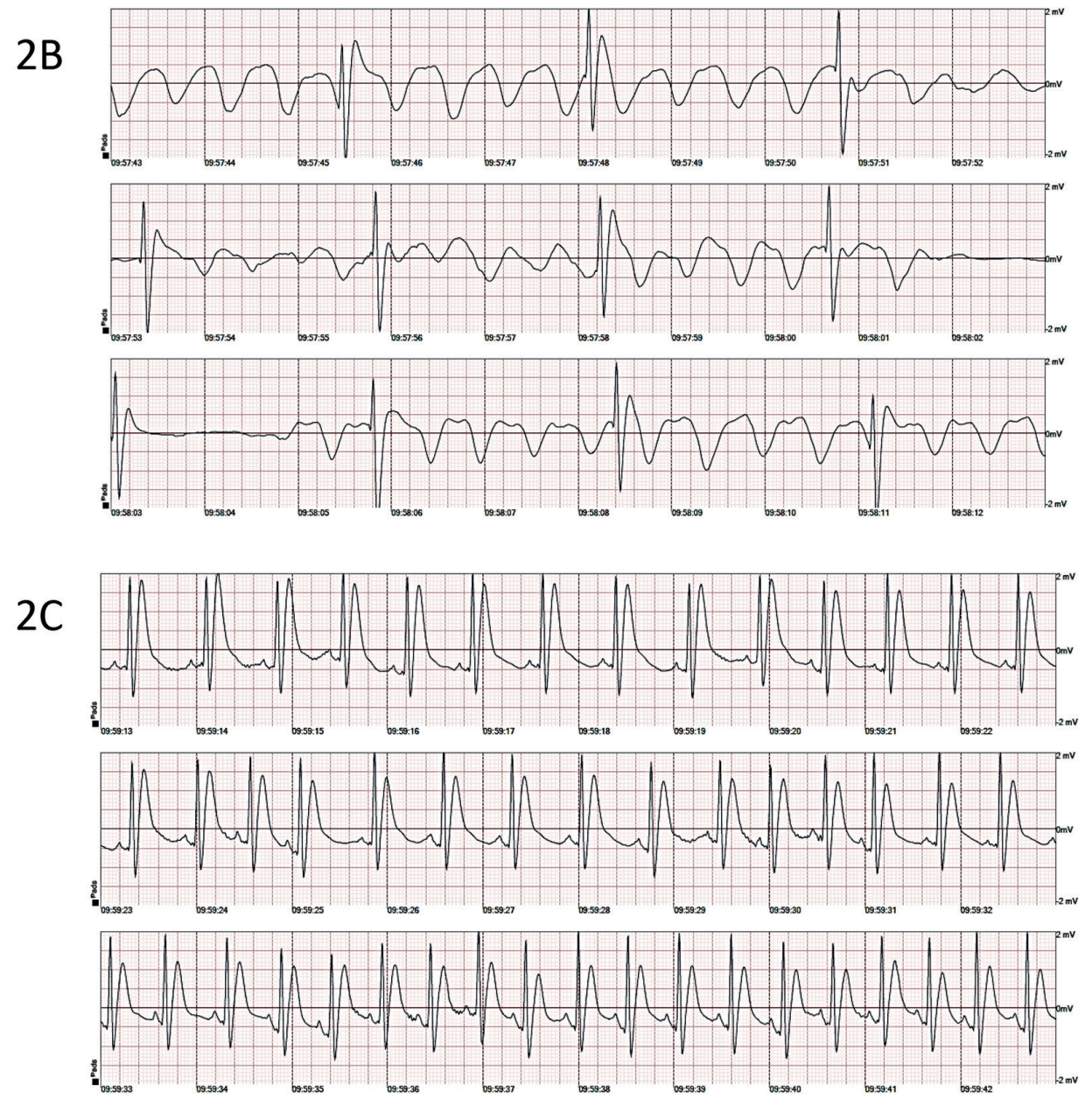

Figura 2. Registro electrocardiográfico del DEA durante paro cardiorrespiratorio. A: Registro FV; en círculo: desfibrilación con 150 J y posterior asistolia. B: Ritmo ventricular durante RCPB. C: Ritmo sinusal. 

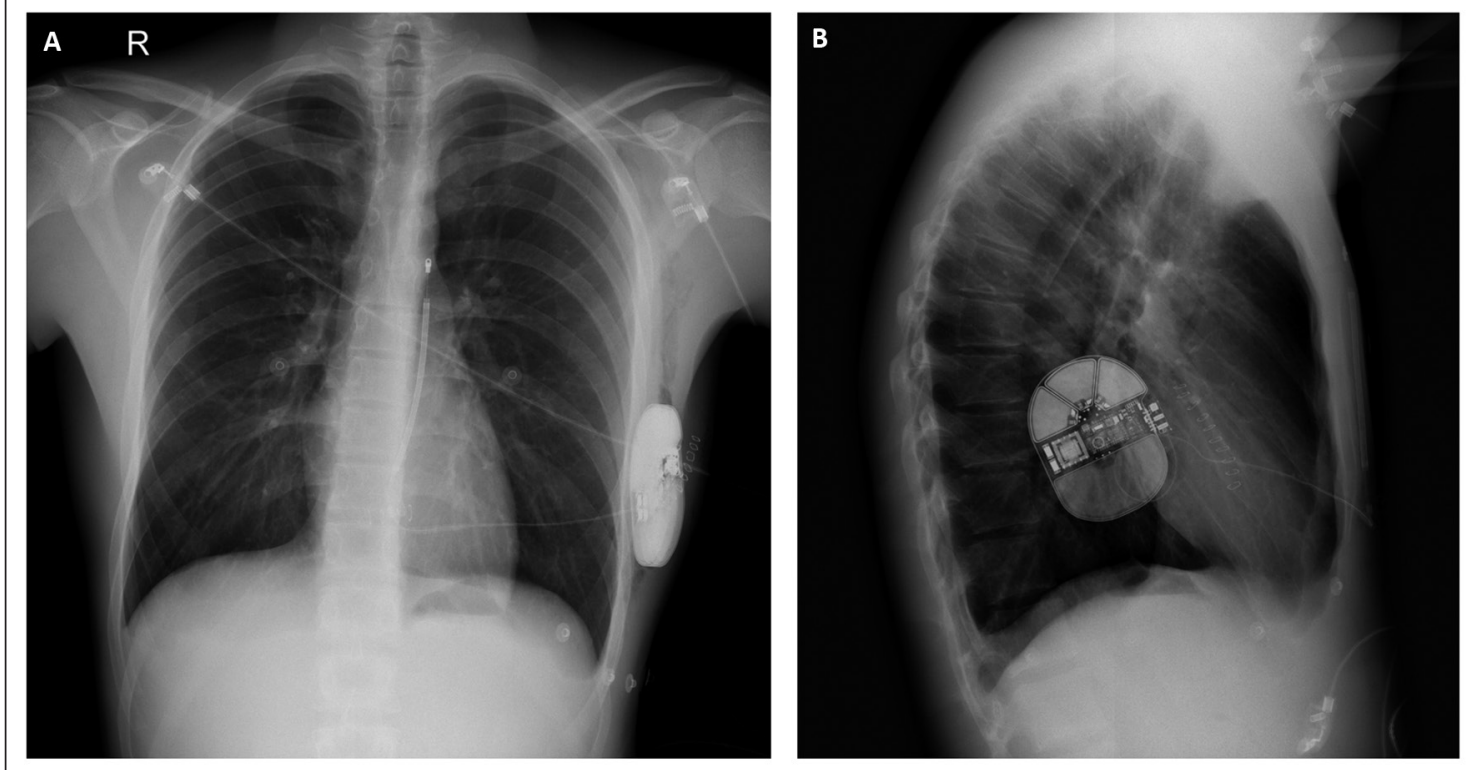

Figura 3. Rx de tórax AP y Lat. muestra DAI Sbc en región axilar izquierda y electrodo a nivel del borde paraesternal izquierdo. A: Proyección AP. B: Proyección Lat.

\section{Discusión}

Indudablemente el ejercicio físico mejora calidad de vida, evita sobrepeso, reduce presión arterial, mejora el perfil lipídico y la insulina resistencia, disminuyendo morbimortalidad por enfermedad coronaria ${ }^{4}$.

Sin embargo, el ejercicio extremo del deporte de resistencia, como ciclismo, maratón y triatlón, puede ser deletéreo, a través de intensa estimulación vagal e inducción de sustrato arritmogénico en aurícula y ventrículo, generando fibrilación auricular, enfermedad del sistema excitoconductor, y TV maligna, responsable de MSC ${ }^{4}$, más aún si existe cardiopatía estructural o una canalopatía oculta, constituyendo muchas veces la única, primera y última manifestación de la enfermedad $^{4-6}$. De ahí la importancia de una completa evaluación cardiológica predeportiva para evitar una catástrofe $\mathrm{f}^{4-6}$.

En España ${ }^{11,12}$, fútbol, ciclismo y atletismo (carreras) son los deportes más frecuentemente asociados a MSC, atribuida a canalopatía en 6\% de los $\operatorname{casos}^{12}$.

Por cada minuto de retraso en la desfibrilación, la sobrevida disminuye en $12 \%{ }^{1}$, con una sobrevida de $25 \%$ si la RCP se inicia en los primeros tres minutos, bajando a 3\%, si es después del tercero ${ }^{10}$. La clave del éxito se basa en el inicio inmediato de RCPB y desfibrilación con DEA, por un equipo entrenado y presente en el evento deportivo ${ }^{1,10}$, como ocurrió en nuestro caso.

En el SBr existe disfunción de los canales de sodio, es de herencia autosómica dominante, representando 4 a 12\% de todas las causas de MSC y $20 \%$ de las que ocurren sin cardiopatía estructural, afectando preferentemente a hombres en la cuarta década de la vida, con una prevalencia de 1-5/10.000 habitantes en Europa y en sudeste asiático 12/10.000 habitantes ${ }^{8}$.

La reducción en la corriente de sodio por mutación del gen SCN5A que se detecta en 20$30 \%$ de los casos, produce un predominio de las corrientes de eflujo de potasio (Ito) sobre corrientes de influjo (INa e ICa), con pérdida del domo del potencial de acción en las células epicárdicas, generando gradiente de voltaje transmural con el endocardio, heterogeneidad en la refractariedad de las distintas capas, favoreciendo mecanismos de reentrada y arritmias ventriculares polimorfas ${ }^{8}$.

Esta anomalía del potencial de acción es responsable del pBr tipo I con elevación descendente del segmento $\mathrm{ST} \geq 2 \mathrm{~mm}$, seguido de onda $\mathrm{T}$ 
negativa en a lo menos una derivación de V1 a V3, siendo el único y definitivo criterio electrocardiográfico ${ }^{8}$. Ocasionalmente es difícil diferenciar el $\mathrm{pBr}$ del remodelamiento eléctrico no patológico del del atleta, caracterizado por BIRD, elevación del segmento ST, repolarización precoz y onda T negativa en derivaciones precordiales ${ }^{13}$.

Dado que el pBr I al ECG puede ser variable en el tiempo y transitoriamente normal en pacientes con SBr, la prueba con Ajmalina, potente bloqueador del canal de sodio, de muy rápida acción, en dosis de $1 \mathrm{mg} / \mathrm{kilo}$ en $5 \mathrm{~min}$ vía ev, permite desenmascarar el pBr I, y en pacientes con mutación genética SC5NA identificada, confirmar diagnóstico, con sensibilidad de $80 \%$, especificidad $94 \%$, valor predictivo positivo $93 \%$ y negativo en $83 \%{ }^{8,14}$. En Chile como alternativa a la prueba de Ajmalina disponemos de otros bloqueadores del canal de sodio, como flecainida oral (400 mg por una vez) o procainamida ev $(10 \mathrm{mg} / \mathrm{k}$ en $10 \mathrm{~min})$, pero de menor eficacia diagnóstica ${ }^{8}$.

La MSC como presentación clínica se considera un factor predictivo independiente de tener una respuesta positiva a la Ajmalina, observándose ésta, en $50 \%$ de los pacientes con $\mathrm{SBr}^{14}$. Otros factores son: sexo masculino, historia familiar de $\mathrm{SBr}$, bloqueo AV de I grado, pBr tipo II o en silla de montar, y el bloqueo basal de rama derecha ${ }^{14}$.

Nuestro paciente debutó con FV, tenía BIRD y prueba con Ajmalina positiva.

Si no se dispone de bloqueadores del canal de sodio, y hay sospecha electrocardiográfica de $\mathrm{SBr}$, la realización de un test de esfuerzo (TE) permite evidenciar un $\mathrm{pBr}$ I, transitoriamente en el peak del ejercicio $^{15}$, pero de preferencia en los primeros tres minutos de etapa de recuperación, por desbalance autonómico con predominio parasimpático vagal, hallazgo que constituiría un factor independiente de riesgo de $\mathrm{FV}$, principalmente en paciente que se presenta asintomático o con síncope $e^{9,16-18}$.

Anecdóticamente se han descrito episodios de arritmias ventriculares en TE de pacientes con $\mathrm{SBr}^{9}$, sin embargo, en la práctica clínica no hay clara relación entre ejercicio y riesgo de MSC en el atleta con $\mathrm{SBr}^{13,17}$.

De hecho, el atleta con pBr tipo I asintomático, puede participar en actividad deportiva que no implique tener alza térmica $\geq 39^{\circ} \mathrm{C}^{13}$, condición ambiental que puede presentarse en ambientes muy calurosos, asociados a deportes extremos.

El alza térmica que inactiva los canales de sodio y la acetilcolina de la hipertonía vagal que bloquea los canales de calcio ${ }^{8}$, al acentuar la expresión fenotípica, se postulan como factores desencadenantes de arritmias ventriculares en el atleta con $\mathrm{SBr}^{9,13}$.

El DAI es el tratamiento más efectivo para prevención secundaria de MSC en atleta con $\mathrm{SBr}^{2,8,11,13}$. En registro español de DAI del 2017, el SBr originó $2,4 \%$ de los primoimplantes ( $16 \%$ por MS recuperada), después de cardiopatía isquémica $53 \%$, miocardiopatía dilatada $26 \%$ e hipertrófica $6,6 \%{ }^{19}$.

Los DAI Sbc en ese año correspondieron a $5,3 \%$ del total de dispositivos implantados ${ }^{19}$.

En SBr el DAI no es contraindicación absoluta para la práctica deportiva sin impacto, no competitiva ${ }^{13}$.

En el joven, sin cardiopatía estructural, sin necesidad de estimulación por bradicardia ni de sobreestimulación para convertir TV, el DAI $\mathrm{Sbc}$ es una alternativa de no inferior eficacia en relación a DAI intracardíaco, pero sin los riesgos de neumotórax, perforación, desplazamiento de cables, trombosis y endocarditis, propios del dispositivo intracardíaco ${ }^{2,20,21}$.

Agradecimientos: Al Dr. B. Kuzmicic, Hospital Dipreca, por facilitarnos la Ajmalina.

\section{Referencias}

1. Delhomme C, Njeim M, Varlet E, Pechmajou L, Benameur N, Cassan P, et al. Automated external defibrillator use in out-of-hospital cardiac arrest: Current limitations and solutions. Arch Cardiovasc Dis. 2019; 112 (3): 217-22. doi: 10.1016/j.acvd.2018.11.001. Epub 2018 Dec 26. PMID: 30594573.

2. Al-Khatib SM, Stevenson WG, Ackerman MJ, Bryant WJ, Callans DJ, Curtis AB, et al. 2017 AHA/ACC/HRS guideline for management of patients with ventricular arrhythmias and the prevention of sudden cardiac death. Circulation. 2018, 138(13): e272-391.

3. Pelliccia A, Sharma S, Gati S, Bäck M, Börjesson M, Caselli S, et al. ESC Scientific Document Group. Eur Heart J. 2020 Aug 29:ehaa 605. doi: 10.1093/eurheartj/ ehaa605. Online ahead of print. PMID:32860412

4. Sharma S, Merghani A, Mont L. Exercise and the heart: the good, the bad, and the ugly. Eur Heart J. 2015 Jun 14; 36 (23): 1445-53. doi: 10.1093/eurheartj/ehv090. Epub 2015 Apr 2. PMID: 25839670. 
5. Corrado D, Zorzi A. Sudden death in athletes. Int J Cardiol. 2017 Jun 15; 237:67-70. doi: 10.1016/j.ijcard.2017.03.034. Epub 2017 Mar 10. PMID: 28318658.

6. Asif IM, Harmon KG. Incidence and Etiology of Sudden Cardiac Death: New Updates for Athletic Departments. Sports Health. 2017; 9 (3): 268-79. doi: 10.1177/1941738117694153. Epub 2017 Feb 1. PMID: 28452637 ; PMCID: PMC5435153.

7. Huikuri HV, Castellanos A, Myerburg RJ. Sudden death due to cardiac arrhythmias. N Engl J Med. 2001; 345 (20): 1473-82. doi: 10.1056/NEJMra000650. PMID: 11794197.

8. Benito B, Brugada J, Brugada P. Síndrome de Brugada. Rev Esp Cardiol. 2009: 62 (11): 1297-315.

9. Batra AS, Watson R, McCanta AC. Exercise-induced syncope and Brugada syndrome. Ann Pediatr Cardiol. 2019; 12 (3): 292-4. doi: 10.4103/apc.APC_131_18. PMID: 31516285 ; PMCID: PMC6716303.

10. DeFroda SF, McDonald C, Myers C, Cruz AI, Owens BD, Daniels AH. Sudden Cardiac Death in the Adolescent Athlete: History, Diagnosis, and Prevention. Am J Med. 2019; 132 (12): 1374-80. doi: 10.1016/j.amjmed.2019.05.025. Epub 2019 Jun 12. PMID: 31199891.

11. Manonelles P, Aguilera B, Boraita A, Luengo E, Pons de Beristain C, Suárez MP. La muerte súbita en el deporte. Registro en el Estado español. Apunts Medicina de L'esport 2007; 153: 26-35.

12. Morentin B, Suárez-Mier MP, Monzó A, Ballesteros J, Molina P, Lucena J. Sports-related sudden cardiac death in Spain. A multicenter, population-based, forensic study of 288 cases. Rev Esp Cardiol (Engl Ed). 2020 Sep 9: S1885-5857(20)30356-X. English, Spanish. doi: 10.1016/j.rec.2020.05.044. Epub ahead of print. PMID: 32920001 .

13. Heidbuchel H, Arbelo E, D’Ascenzi F, Borjesson M, Boveda S, Castelletti S, et al. Recommendations for participation in leisure-time physical activity and competitive sports of patients with arrhythmias and potentially arrhythmogenic conditions. Part 2: ventricular arrhythmias, channelopathies, and implantable defibrillators. Europace. 2020 Jun 29: euaa106. doi: 10.1093/europace/ euaa106. Epub ahead of print. PMID: 32596731.

14. Veltmann C, Wolpert C, Sacher F, Mabo P, Schimpf
R, Streitner F, et al. Response to intravenous ajmaline: a retrospective analysis of 677 ajmaline challenges. Europace. 2009; 11 (10): 1345-52. doi: 10.1093/europace/ eup189. Epub 2009 Jul 9. PMID: 19589796.

15. Tijskens M, Heidbuchel H, Sarkozy A. Supranormal heart rate during peak exercise stress test triggering type-1 Brugada ECG pattern. J Cardiovasc Electrophysiol 2019; 30: 1367-68. https://doi.org/10.1111/ jce.13973.

16. Amin AS, de Groot EA, Ruijter JM, Wilde AA, Tan HL. Exercise-induced ECG changes in Brugada syndrome. Circ Arrhythm Electrophysiol. 2009; 2 (5): 531-9. doi: 10.1161/CIRCEP.109.862441. Epub 2009 Aug 24. PMID: 19843921.

17. Mascia G, Arbelo E, Hernández-Ojeda J, Solimene F, Brugada R, Brugada J. Brugada Syndrome and Exercise Practice: Current Knowledge, Shortcomings and Open Questions. Int J Sports Med. 2017; 38 (8): e2. doi: 10.1055/s-0043-120577. Epub 2018 Jan 23. Erratum for: Int J Sports Med. 2017; 38 (8): 573-81. PMID: 29361645.

18. Marrakchi S, Kammoun I, Bennour E, Laroussi L, Ben Miled M, Kachboura S. Inherited primary arrhythmia disorders: cardiac channelopathies and sports activity. Herz. 2020; 45 (2): 142-57. English. doi: 10.1007/ s00059-018-4706-2. Epub 2018 May 9. PMID: 29744527.

19. Fernández Lozano I, Osca Asensi J, Alzueta Rodríguez J. Registro Español de Desfibrilador Automático Implantable. XV Informe Oficial de la Sección de Electrofisiología y Arritmias de la Sociedad Española de Cardiología (2018). Rev Española Cardiología 2019; 72 (12): 105464, DOI: 10.1016/j.recesp.2019.07.029.

20. Bogush N, Espinosa RE, Cannon BC, Wackel PL, Okamura H, Friedman PA, et al. Selecting the right defibrillator in the younger patient: Transvenous, epicardial or subcutaneous? Int J Cardiol. 2018; 250: 133-8. doi: 10.1016/j.ijcard.2017.09.213. Epub 2017 Oct 13. Erratum in: Int J Cardiol. 2018 Mar 8;: PMID: 29169751.

21. Knops R, Olde Nordkamp L, Delnoy P, Boersma L, Kuschyk J, El-Chami M et al. Subcutaneous or Transvenous Defibrillator Therapy. N Engl J Med 2020; 383: 526-36. DOI: 10.1056/NEJMoa1915932. 